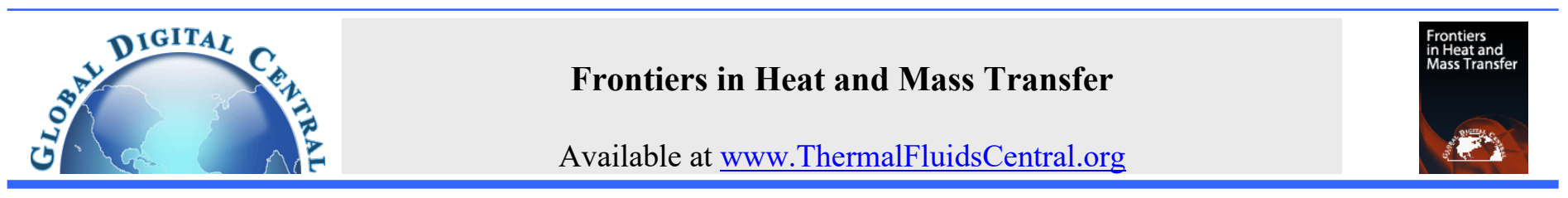

\title{
TURBULENT HEAT TRANSFER IN AN AXIALLY ROTATING PIPE AT HIGH ROTATION RATE: A NUMERICAL STUDY
}

\author{
Obed Y.W. Abotsi, John P. Kizito* \\ Department of Mechanical Engineering, North Carolina A\&T State University, Greensboro, NC, 27411, USA
}

\begin{abstract}
In this paper, turbulent water flow and heat transfer are studied numerically in a pipe which is rotating about its longitudinal axis. Computations were conducted for axial Reynolds numbers ranging from 10000 to 30000 at different rotation rates. Rotation rate $(N)$ is the ratio of the rotational Reynolds number to the axial Reynolds number. Predictions showed that the Nusselt number $(N u)$ of the stationary pipe $(N=0)$ was augmented by $50-58 \%$ at $N=5,105-132 \%$ at $N=10,150-201 \%$ at $N=15,208-265 \%$ at $N=20$, and $320-373 \%$ at $N=30$. Improvements in the heat transfer rate was linked to the introduction of tangential velocity components from the moving pipe wall which created secondary flow.

Keywords: Rotation rate, Nusselt number, heat transfer, secondary flow
\end{abstract}

\section{INTRODUCTION}

Turbulent flow and heat transfer in rotating channels are encountered in numerous industrial applications such as flow in the inlet part of fluid machines (Nishibori et al., 1987), cooling system of rotors (Imao et al., 1992) combustion chambers (Satake et al., 2002) and rotating heat pipes (Faghri et al., 1993; Lian et al., 2020). Many theoretical, experimental, and numerical studies have been performed to investigate the influence of rotation on the flow and heat transfer. However, these investigations are limited to low rotation rates. Rotation rate $(N)$ is the ratio of the rotational Reynolds number $\left(R e_{\Omega}\right)$ to the axial Reynolds number $\left(R e_{a}\right)$. Pedley (1969) showed that the Hagen-Poiseuille flow is unstable to infinitesimal disturbances when the pipe rotates about its longitudinal axis. These results have been confirmed with experiments (Nagib et al., 1971) and numerical solutions (Mackrodt, 1976). Borisenko et al. (1973) determined the turbulent velocity fluctuations in a rotating pipe and revealed that they were suppressed by rotation and when the flow advanced downstream, the suppressions extended towards the central section of the pipe. Imao et al. (1996) measured the turbulent fluctuations of flow in an axially rotating pipe at axial Reynolds number of 20000 and rotation rate ranging from 0 to $1(0 \leq \mathrm{N} \leq 1)$. From their experiments, they showed that as the rotation rate increased, the turbulent fluctuations decreased. Numerical investigations by Hirai et al. (1988), Ould-Rouiss et al. (2010) and Granados-Ortiz et al. (2019), also showed that rotation affects the turbulent intensities.

Kikuyama et al. (1983a) measured the time-mean velocities and turbulent fluctuations inside the developed turbulent boundary layer of an axially rotating pipe at $0 \leq \mathrm{N} \leq 1$ using hot-wire probes. They showed that rotation produces two opposite effects on the flow: a destabilizing effect due to large shear caused by the moving pipe wall which prevails in the inlet region, and a stabilizing effect induced by the swirling velocity component which becomes dominant in the downstream section. By measuring the velocity distribution at various axial positions from the entrance, Murakami and Kikuyama (1980) studied the flow pattern in an axially rotating pipe at $0 \leq \mathrm{N} \leq 3$. They observed that with an increase in the rotation rate the axial velocity profile approaches the HaganPoiseuille flow. Kikuyama et al. (1983b) applied the correlation between the mixing length and Richardson number proposed by Bradshaw (1969) to study the flow structure in a pipe for rotation rates between 0 and 3 . They demonstrated that rotation can cause an existing turbulent flow to reverse back to laminar flow (laminarization). Nishibori et al. (1987) examined the laminarization phenomenon of a fully developed turbulent flow in the inlet region of an axially rotating pipe. Their results revealed that flow laminarization was significant in the inlet region where a nonrotating inner core existed and as the flow proceeded downstream, bursts of turbulence appeared and the laminarized flow became unstable.

The influence of rotation on the hydraulic resistance has been experimentally investigated by White (1964) and Shchukin (1967). If the approaching flow is turbulent, the pressure loss decreases with the increase of the rotational speed of the pipe. Canon and Kays (1969) performed experiments to study heat transfer in an axially rotating pipe. The most noticeable effect of rotation was observed in the transition region (from laminar to turbulent) and the convective heat transfer was insensitive to the rotation once the flow became fully turbulent. Visualization of flow revealed that rotation stabilizes laminar flow to an extent that transition occurs at higher axial Reynolds numbers. Reich and Beer (1989) carried out experimental and analytical studies to determine the velocity distribution and heat transfer in an axially rotating pipe. They modified the mixing length model proposed by Kikuyama et al. (1983b) for their analytical study. For turbulent axial flow at $0 \leq \mathrm{N} \leq 5$, they showed that increasing the rotational speed of the pipe decreased the heat transfer. Reduction in the heat transfer and friction coefficient was caused by radial suppressions due to the presence of centrifugal forces. In their paper, Weigand and Beer (1992) studied the effect of rotation on the heat transfer of fluid flowing in a rotating pipe at $0 \leq \mathrm{N} \leq 5$. They showed that flow laminarization caused a reduction in heat transfer. However, it was noted that these investigations were only valid for large geometric aspect ratios. 
For short pipes, Seghir-Ouali et al. (2006) studied the influence of rotation on the heat transfer rate in an axially rotating pipe at speeds ranging from 4 to $880 \mathrm{rpm}$. They showed from their experiments that the convective heat transfer coefficient increased with the rise of the rotational speed. Chong (2015) examined the heat transfer and pressure loss associated with axial airflow in the rotor-stator gap at $0 \leq \mathrm{N} \leq 6$. He concluded that increasing the rotation rate of the pipe increased both the Nusselt number and pressure drop. The rotational pressure loss accounted for approximately $50 \%$ of the total system loss. CFD simulations carried out by Gai et al. (2018) at $0 \leq \mathrm{N} \leq 2.5$ indicated that the pressure loss in a rotating pipe increased with increasing rotation rate. Other researchers have also shown that rotation enhances the rate of heat transfer (Staton and Cavagnino, 2008; Hettegger et al.,2011; and Gai et al., 2017). To the author's knowledge, turbulent liquid flow and heat transfer in an axially rotating pipe at very high rotation rates has never been studied.

The objective of the present work is to investigate the effect of high rotation rate on the heat transfer and pressure loss in an axially rotating.

\section{PROBLEM FORMULATION}

When fluid enters an axially rotating pipe, it receives a tangential component of velocity from the moving wall which causes the fluid to rotate with the pipe (Weigand and Beer, 1994). This creates a density gradient that drives relatively less dense and warmer fluid at the wall to move inwards and for denser and colder fluid at the core region of the pipe to move outwards. Interactions between the density gradient and the Coriolis and centrifugal forces yield a complex secondary flow. Consequently, equations that describe flow in stationary pipes cannot be used to analyze flow in rotating pipes.

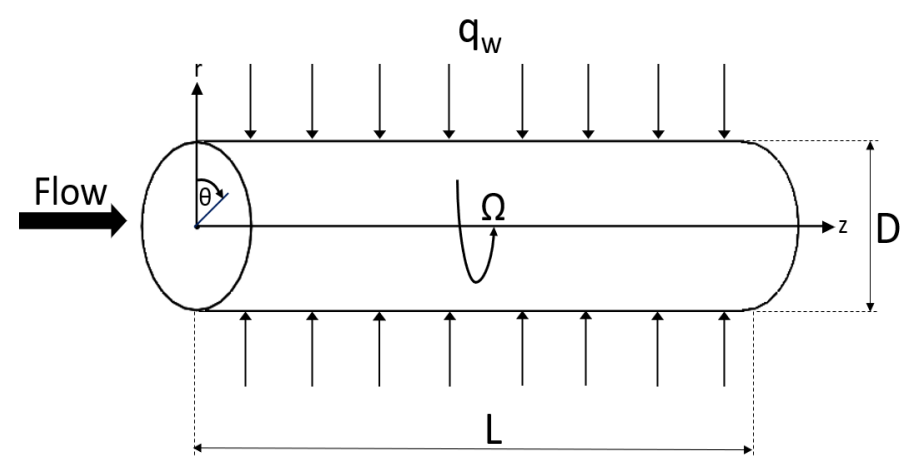

Fig. 1 Schematic of the model

In the present study, the fluid flow and heat transfer processes are described using three conservation laws in the form of differential equations. Fig. 1 depicts a horizontal pipe rotating about the $\mathrm{z}$-axis with constant angular velocity, $\Omega$. The incompressible Newtonian fluid is heated by imposing a uniform heat flux, $\mathrm{q}_{\mathrm{w}}$, on the pipe wall. It is assumed that the flow is steady and the thermophysical properties of the working fluid except for density are independent of temperature. Considering the Boussinesq approximation and neglecting viscous dissipation, the basic equations that describe the flow are given as:

\subsection{Continuity Equation}

$\frac{V_{r}}{r}+\frac{\partial V_{r}}{\partial r}+\frac{\partial V_{z}}{\partial z}+\frac{1}{r} \frac{\partial V_{\theta}}{\partial \theta}=0$

where $V_{r}, V_{\theta}$, and $V_{z}$ are the components of velocity in the directions of $r, \theta$, and $z$ respectively.

\subsection{Momentum Equation}

$r$-component

$\rho\left(V_{r} \frac{\partial V_{r}}{\partial r}+\frac{V_{\theta}}{r} \frac{\partial V_{r}}{\partial \theta}-\frac{V_{\theta}^{2}}{r}+V_{z} \frac{\partial V_{r}}{\partial z}\right)=-\frac{\partial P}{\partial r}+\rho g \beta\left(T-T_{\infty}\right)+$ $\rho\left(\Omega^{2} r+2 \Omega V_{\theta}\right) \beta\left(T-T_{\infty}\right)+\mu\left(\frac{\partial^{2} V_{r}}{\partial r^{2}}+\frac{1}{r} \frac{\partial V_{r}}{\partial r}-\frac{V_{r}}{r^{2}}+\frac{1}{r^{2}} \frac{\partial^{2} V_{r}}{\partial \theta^{2}}-\frac{2}{r^{2}} \frac{\partial V_{\theta}}{\partial \theta}+\right.$

$\left.\frac{\partial^{2} V_{r}}{\partial z^{2}}\right)$

$\theta$-component

$\rho\left(V_{r} \frac{\partial V_{\theta}}{\partial r}+\frac{V_{\theta}}{r} \frac{\partial V_{\theta}}{\partial \theta}+\frac{V_{r} V_{\theta}}{r}+V_{z} \frac{\partial V_{\theta}}{\partial z}\right)=-\frac{1}{r} \frac{\partial P}{\partial \theta}-2 \rho \Omega V_{r} \beta\left(T-T_{\infty}\right)+$

$\mu\left(\frac{\partial^{2} V_{\theta}}{\partial r^{2}}+\frac{1}{r} \frac{\partial V_{\theta}}{\partial r}-\frac{V_{\theta}}{r^{2}}+\frac{1}{r^{2}} \frac{\partial^{2} V_{\theta}}{\partial \theta^{2}}+\frac{2}{r^{2}} \frac{\partial V_{r}}{\partial \theta}+\frac{\partial^{2} V_{\theta}}{\partial z^{2}}\right)$

$z$-component

$$
\rho\left(V_{r} \frac{\partial V_{z}}{\partial r}+\frac{V_{\theta}}{r} \frac{\partial V_{z}}{\partial \theta}+V_{z} \frac{\partial V_{z}}{\partial z}\right)=-\frac{\partial P}{\partial z}+\mu\left(\frac{\partial^{2} V_{z}}{\partial r^{2}}+\frac{1}{r} \frac{\partial V_{z}}{\partial r}+\frac{1}{r^{2}} \frac{\partial^{2} V_{z}}{\partial \theta^{2}}+\frac{\partial^{2} V_{z}}{\partial z^{2}}\right)
$$

where $P$ is the pressure, $g$ is the acceleration due to gravity, $\rho$ is density, $\mu$ is the dynamic viscosity, $\beta$ is the coefficient of thermal expansion, and $\mathrm{T}$ and $\mathrm{T}_{\infty}$ are the temperature and free stream temperature respectively.

\subsection{Energy Equation}

$\rho C_{p}\left(V_{r} \frac{\partial T}{\partial r}+\frac{V_{\theta}}{r} \frac{\partial T}{\partial \theta}+V_{z} \frac{\partial T}{\partial z}\right)=k\left[\frac{\partial^{2} T}{\partial r^{2}}+\frac{1}{r} \frac{\partial T}{\partial r}+\frac{1}{r^{2}} \frac{\partial^{2} T}{\partial \theta^{2}}+\frac{\partial^{2} T}{\partial z^{2}}\right]$

where $C p$ is the specific heat at constant pressure and $k$ is the thermal conductivity.

\subsection{Dimensionless Variables}

To obtain a system of non-dimensionalized equations, the following dimensionless variables are introduced:

$$
\begin{array}{lll}
r^{*}=\frac{r}{\frac{1}{2} D}, & z^{*}=\frac{z}{L} & T^{*}=\frac{T-T_{\infty}}{T_{s}-T_{\infty}} \\
V_{r}{ }^{*}=\frac{V_{r}}{\frac{1}{2} \Omega D} & V_{\theta}{ }^{*}=\frac{V_{\theta}}{\frac{1}{2} \Omega D}, & V_{z}^{*}=\frac{V_{z}}{V_{m}} \\
P^{*}=\frac{P}{\frac{1}{4} \rho \Omega^{2} D^{2}} &
\end{array}
$$

where $D$ and $L$ are the diameter and length of the pipe respectively and $V_{m}$ is the mean axial velocity. The dimensionless form of the basic flow equations are presented below:

\subsection{Dimensionless Continuity Equation}

$\frac{V_{r}^{*}}{r^{*}}+\frac{\partial V_{r}^{*}}{\partial r^{*}}+\frac{1}{N(L / D)} \frac{\partial V_{z}^{*}}{\partial z^{*}}+\frac{1}{r^{*}} \frac{\partial V_{\theta}^{*}}{\partial \theta^{*}}=0$

\subsection{Dimensionless Momentum Equation}

$r$-component

$$
\begin{aligned}
& V_{r}^{*} \frac{\partial V_{r}^{*}}{\partial r^{*}}+\frac{V_{\theta}^{*}}{r^{*}} \frac{\partial V_{r}^{*}}{\partial \theta^{*}}-\frac{V_{\theta}^{* 2}}{r^{*}}+\frac{1}{N(L / D)} V_{z}^{*} \frac{\partial V_{r}^{*}}{\partial z^{*}}=-\frac{\partial P^{*}}{\partial r^{*}}+\frac{G r_{g}}{R e_{\Omega}^{2}} T^{*}+\left(r^{*}+\right. \\
& \left.2 V_{\theta}^{*}\right) \frac{G r_{\Omega}}{R e_{\Omega}^{2}} T^{*}+\frac{4}{R e_{\Omega}}\left(\frac{\partial^{2} V_{r}^{*}}{\partial r^{* 2}}+\frac{1}{r^{*}} \frac{\partial V_{r}^{*}}{\partial r^{*}}-\frac{V_{r}^{*}}{r^{* 2}}+\frac{1}{r^{* 2}} \frac{\partial^{2} V_{r}^{*}}{\partial \theta^{* 2}}-\frac{2}{r^{* 2}} \frac{\partial V_{\theta}^{*}}{\partial \theta^{*}}+\right. \\
& \left.\frac{1}{4(L / D)^{2}} \frac{\partial^{2} V_{r}^{*}}{\partial z^{* 2}}\right)
\end{aligned}
$$


$\theta$-component

$V_{r}^{*} \frac{\partial V_{\theta}^{*}}{\partial r^{*}}+\frac{V_{\theta}^{*}}{r^{*}} \frac{\partial V_{\theta}^{*}}{\partial \theta^{*}}+\frac{V_{r}^{*} V_{\theta}^{*}}{r^{*}}+\frac{1}{N(L / D)} V_{z}^{*} \frac{\partial V_{\theta}^{*}}{\partial z^{*}}=-\frac{1}{r^{*}} \frac{\partial P^{*}}{\partial \theta^{*}}-2 V_{r}^{*} \frac{G r_{\Omega}}{R e_{\Omega}^{2}} T^{*}+$

$\frac{4}{R e_{\Omega}}\left(\frac{\partial^{2} V_{\theta}{ }^{*}}{\partial r^{* 2}}+\frac{1}{r^{*}} \frac{\partial V_{\theta}{ }^{*}}{\partial r^{*}}-\frac{V_{\theta}^{*}}{r^{* 2}}+\frac{1}{r^{* 2}} \frac{\partial^{2} V_{\theta}{ }^{*}}{\partial \theta^{* 2}}+\frac{2}{r^{* 2}} \frac{\partial V_{r}^{*}}{\partial \theta^{*}}+\frac{1}{4(L / D)^{2}} \frac{\partial^{2} V_{\theta}{ }^{*}}{\partial z^{* 2}}\right)$

$z$-component

$V_{r}^{*} \frac{\partial V_{Z}{ }^{*}}{\partial r^{*}}+\frac{V_{\theta}^{*}}{r^{*}} \frac{\partial V_{Z}{ }^{*}}{\partial \theta^{*}}+\frac{1}{N(L / D)} \frac{\partial V_{Z}{ }^{*}}{\partial z^{*}}=-\frac{1}{4} \frac{N}{(L / D)} \frac{\partial P^{*}}{\partial z^{*}}+\frac{4}{R e_{\Omega}}\left(\frac{\partial^{2} V_{Z}{ }^{*}}{\partial r^{* 2}}+\frac{1}{r^{*}} \frac{\partial V_{Z}{ }^{*}}{\partial r^{*}}+\right.$

$\left.\frac{1}{r^{* 2}} \frac{\partial^{2} V_{z}^{*}}{\partial \theta^{* 2}}+\frac{1}{4(L / D)^{2}} \frac{\partial^{2} V_{z}^{*}}{\partial z^{* 2}}\right)$

\subsection{Dimensionless Energy Equation}

$V_{r}^{*} \frac{\partial T^{*}}{\partial r^{*}}+\frac{V_{\theta}^{*}}{r^{*}} \frac{\partial T^{*}}{\partial \theta^{*}}+\frac{1}{N(L / D)} V_{z}^{*} \frac{\partial T^{*}}{\partial z^{*}}=\frac{4}{P e}\left[\frac{\partial^{2} T^{*}}{\partial r^{* 2}}+\frac{1}{r^{*}} \frac{\partial T^{*}}{\partial r^{*}}+\frac{1}{r^{* 2}} \frac{\partial^{2} T^{*}}{\partial \theta^{* 2}}+\right.$ $\left.\frac{1}{4(L / D)^{2}} \frac{\partial^{2} T^{*}}{\partial z^{* 2}}\right]$

From Equations (6) - (10), it can be inferred that flow through an axially rotating pipe is affected by the following parameters:

$R e_{a}=\frac{V_{m} D}{v} ;$ axial Reynolds number

$R e_{\Omega}=\frac{\Omega D^{2}}{v} ;$ rotational Reynolds number

$N=\frac{R e_{\Omega}}{R e_{a}} ;$ rotation rate

$G r_{g}=\frac{g \beta\left(T_{s}-T_{\infty}\right) D^{3}}{v^{2}} ;$ gravitational Grashof number

$G r_{\Omega}=\frac{\Omega^{2} \beta\left(T_{S}-T_{\infty}\right) D^{4}}{v^{2}} ;$ rotational Grashof number

$P e=\operatorname{Re}_{\Omega} \operatorname{Pr} ;$ Peclet number

$\operatorname{Pr}=\frac{C_{p} \mu}{k} ;$ Prandtl number

$L / D ;$ Aspect ratio

The Nusselt number and friction factor can be computed as:

$N u_{x}=\frac{h_{x} D}{k} ;$ local Nusselt Number

$N u=\frac{\int N u_{x} d A_{s}}{\int d A_{s}} ;$ average Nusselt Number

$f=\left(\frac{1}{L / D}\right)\left(\frac{2 \Delta P}{\rho V_{m}^{2}}\right)$

where $h_{x}$ is the convective heat transfer coefficient and $d A_{s}$ is the infinitesimal surface area of the wall.

\section{NUMERICAL PROCEDURE}

Three-dimensional $(3 D)$ numerical simulations are processed with the commercial finite volume software ANSYS Fluent 19.2. The realizable $\mathrm{k}-\varepsilon$ turbulence model is used to provide closure to the Reynolds Average
Navier-Stokes (RANS) equations. The pressure-velocity coupling in the equations is handled with the Coupled algorithm and the convective terms are discretized with the second-order upwind scheme. The single reference frame approach is used to model the rotating domain. Gravity is included in the numerical study and the effect of buoyancy is modeled based on the Boussinesq approximation. The convergent criterion is set as $10^{-6}$ for all scaled residuals.

The pipe has a length of $0.8 \mathrm{~m}$ and a diameter of $0.02 \mathrm{~m}$. A constant heat flux of $10000 \mathrm{~W} / \mathrm{m}^{2}$ and the no-slip condition is specified on the pipe wall. The inlet temperature of flow is set at $300 \mathrm{~K}$. The axial Reynolds numbers which determine the inlet velocity are set at 10000 , 15000, 20000, 25000, and 30000 and investigated at different pipe rotation rates $(0 \leq \mathrm{N} \leq 30)$. The turbulence intensity at the inlet is kept constant at $5 \%$. A pressure outlet condition of zero-gauge pressure is used at the outlet. Water is selected as the working fluid. The dynamic viscosity $(\mu)$, density $(\rho)$, specific heat at constant pressure $(C p)$ and thermal conductivity $(k)$ of water are given as; $\mu=0.00103 \mathrm{~kg} /(\mathrm{m}-\mathrm{s}), \rho=$ $998.2 \mathrm{~kg} / \mathrm{m}^{3}, C p=4182 \mathrm{~J} /(\mathrm{kg}-\mathrm{K})$ and $k=0.6 \mathrm{~W} /(\mathrm{m}-\mathrm{K})$ respectively. The enhanced wall treatment option is used for near-wall modeling of the pipe wall. In each case, the $y+$ value of the wall is less than 0.5 .

A mesh independence study is conducted to ensure that the solution is independent of the grid size. Five grid numbers: 75050, 173482, 233960, 380661, and 497158 are tested for the case where $N=5$ and $R e_{a}=10000$. The variation in the Nusselt number and friction factor between the grid number of 233960 and 497158 was found to be less than $1 \%$. Considering the accuracy of the results and computational time, the mesh with 233960 elements is adopted for calculations. The reliability of the model has been validated by a good agreement between the numerical and experimental results in our previous study (Abotsi and Kizito, 2020).

\section{RESULTS AND DISCUSSION}

CFD results of the velocity and temperature fields of the pipe at different rotation rates are shown in Fig. 2 and Fig. 3. Negative velocities denote flow in the opposite direction. Rotation induces Coriolis and centrifugal acceleration components which yield secondary flow. Secondary flow not only improves radial fluid mixing and boundary layer disturbance but offers a longer pathway for the fluid. It was noticed from Fig. 2 that the strength of secondary flow produced increased with the rise of the rotation rate. Pipe at rotation rate of $5(N=5)$ produced the weakest secondary flow. No secondary flow was produced at $N=0$ (stationary case). The strongest secondary flow was noticed at rotation rate of 30 $(N=30)$. Thus, the velocity of flow in the pipe at rotation rate of 30 is increased and heat dissipates more quickly from the surface.

Fig. 4 depicts the variation of the average Nusselt number $(\mathrm{Nu})$ with the axial Reynolds number $\left(\mathrm{Re}_{\mathrm{a}}\right)$ at different rotation rates of the pipe. It was observed that for all cases the Nusselt number increased with the rise of the axial Reynolds number. Improvement in the heat transfer rate was related to an increase in turbulence as the axial Reynolds number increased which led to the enhancement of the convective heat transfer coefficient. Results from Fig. 4 also revealed a significant improvement in the heat transfer rate with rotation. In comparison to $N=0$ (stationary case), the Nusselt number of the pipe was enhanced by approximately $50-58 \%$ at $N=5,105-132 \%$ at $N=10,150-201 \%$ at $N=15,208-265 \%$ at $N=20$ and $320-373 \%$ at $N=30$. This remarkable enhancement in Nusselt number was attributed to centrifugal buoyancy and Coriolis forces which led to the formation of stronger secondary flows as the rotation rate of the pipe increased. Secondary flow improves flow turbulence and reduces the thickness of the thermal boundary layer. The intensity of secondary flow produced is dependent on the rotating speed of the pipe. Pipe at $N=30$ generated the strongest secondary flow and hence had the best heat transfer rate. 


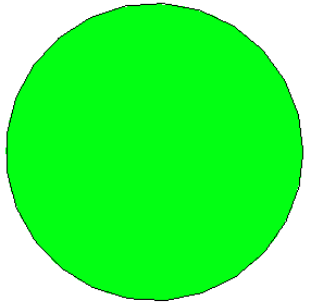

(a) $\mathrm{N}=0$

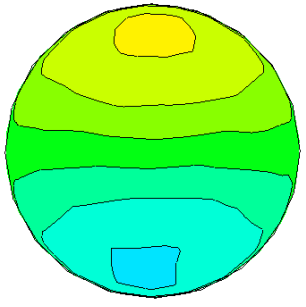

(d) $\mathrm{N}=15$

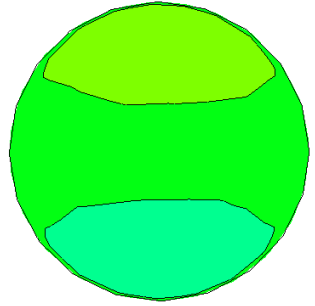

(b) $\mathrm{N}=5$

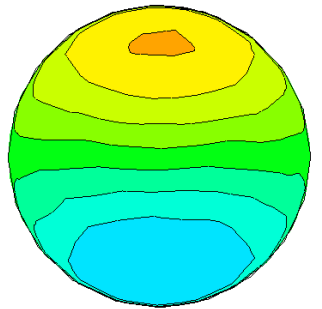

(e) $\mathrm{N}=20$

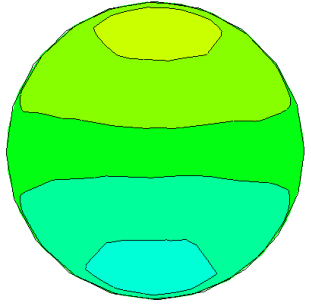

(c) $\mathrm{N}=10$

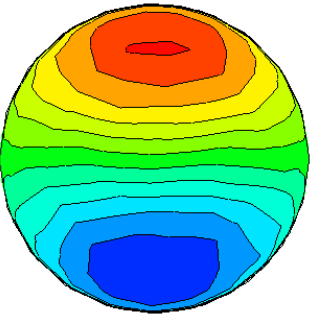

(f) $\mathrm{N}=30$
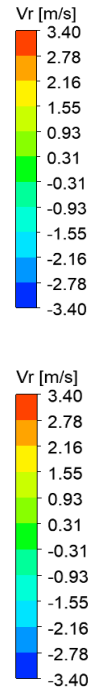

Fig. 2 Contours of radial velocity field at different rotation rate $\left(\mathrm{Re}_{\mathrm{a}}=10000\right)$

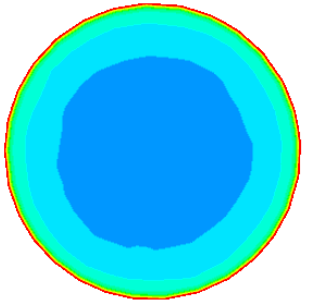

(a) $\mathrm{N}=0$

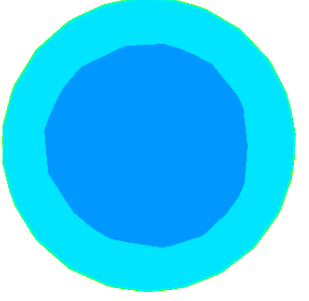

(d) $\mathrm{N}=15$

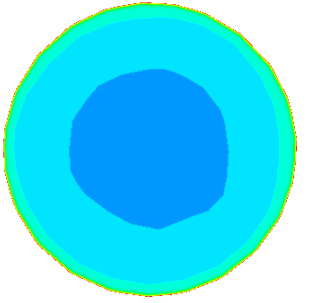

(b) $\mathrm{N}=5$

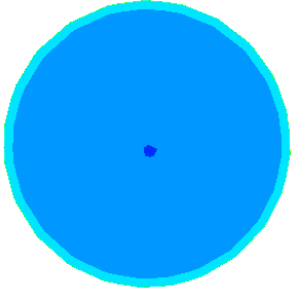

(e) $\mathrm{N}=20$

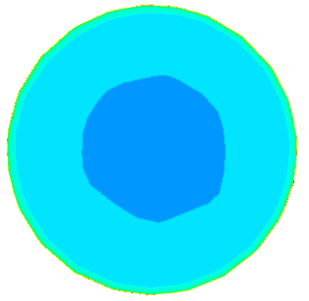

(c) $\mathrm{N}=10$

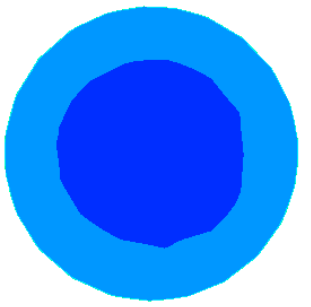

(f) $\mathrm{N}=30$

Temperature $[\mathrm{K}$
\begin{tabular}{|l|}
302.00 \\
301.82 \\
301.64 \\
301.45 \\
301.27 \\
301.09 \\
300.91 \\
300.73 \\
300.55 \\
300.36 \\
300.18 \\
300.00
\end{tabular}

Temperature $[\mathrm{K}$
\begin{tabular}{|l|}
\hline 302.00 \\
301.82 \\
-301.64 \\
301.45 \\
301.27 \\
301.09 \\
300.91 \\
300.73 \\
300.55 \\
300.36 \\
300.18 \\
300.00
\end{tabular}

Fig. 3 Contours of temperature field at different rotation rate $\left(\operatorname{Re}_{\mathrm{a}}=10000\right)$

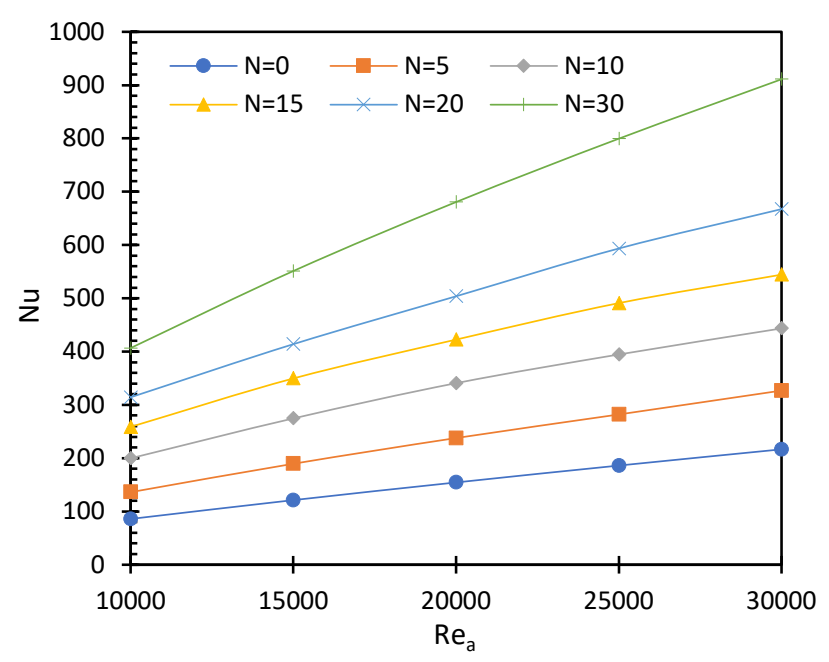

Fig. 4 Variation of Nusselt number with respect to axial Reynolds number at different rotation rate

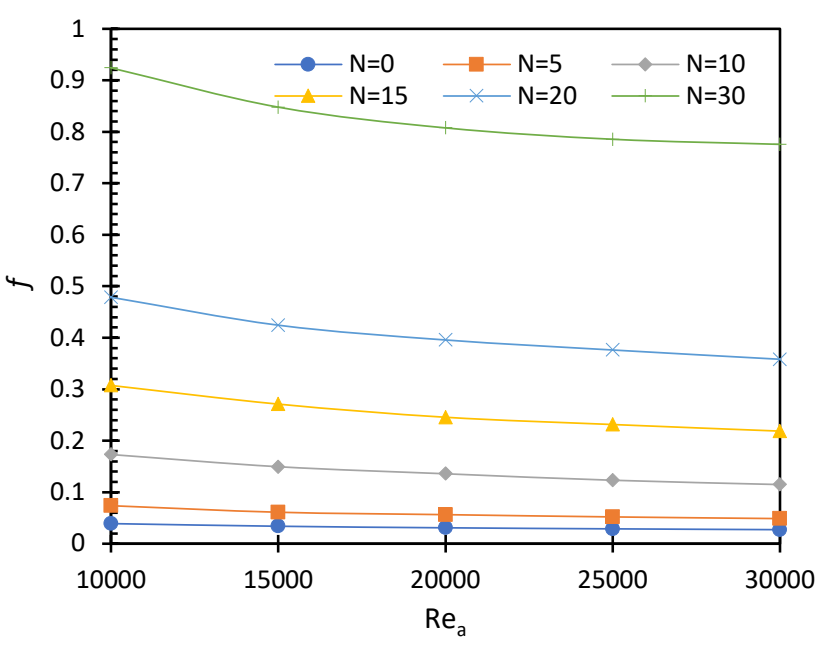

Fig. 5 Variation of friction factor with respect to axial Reynolds number at different rotation rate 
The variation of the friction factor $(f)$ versus the axial Reynolds number $\left(\mathrm{Re}_{\mathrm{a}}\right)$ at different pipe rotation rate are shown in Fig. 5. The friction factor characterizes the hydraulic resistance across the pipe and is relevant in energy consumption. As expected, the friction factor of the rotating pipe was higher than that of the stationary pipe. The calculated friction factor at $\mathrm{N}=5, \mathrm{~N}=10, \mathrm{~N}=15, \mathrm{~N}=20$, and $\mathrm{N}=30$ were in the ranges of 1.82-1.89, 4.30-4.44, 7.87-8.01, 12.25-13.13, and 23.6-26.1 times, respectively, to those obtained in the stationary pipe $(\mathrm{N}=0)$. Increase in the friction factor was linked to the introduction of Coriolis and centrifugal forces from the rotating pipe which increased the wall friction and created large pressure gradients along the radial direction. It was also noticed that increasing the axial Reynolds number reduces the friction factor.

\section{CONCLUSION}

Turbulent water flow and heat transfer in a pipe rotating about its longitudinal axis and under constant wall heat flux boundary condition has been investigated numerically. In the range of axial Reynolds numbers between 10000 and 30000 , the Nusselt number of the pipe at $N=0$ (stationary case) was improved by $50-58 \%$ at $N=5,105-132 \%$ at $N=10,150-201 \%$ at $N=15,208-265 \%$ at $N=20$, and $320-373 \%$ at $N=30$. Enhancement in the heat transfer rate was attributed to the strength of secondary flow generated which intensifies with increasing the rotation rate. The friction factor of the stationary pipe $(\mathrm{N}=0)$ was increased by 1.82-1.89, 4.30-4.44, 7.87-8.01, 12.25-13.13, and 23.6-26.1 times at $N=5, N=10, N=15, N=20$, and $N=30$ respectively.

\section{ACKNOWLEDGMENTS}

We acknowledge the support from NASA NNH16ZEA001N: Electric Propulsion: Challenges and Opportunities.

\section{NOMENCLATURE}

$\begin{array}{ll}\mathrm{A}_{\mathrm{s}} & \left.\text { Infinitesimal area of the wall } \mathrm{m}^{2}\right) \\ \mathrm{Cp} & \text { specific heat at constant pressure } \mathrm{J} /(\mathrm{kg} \mathrm{K}) \\ \mathrm{D} & \text { diameter of the pipe }(\mathrm{m}) \\ f & \text { friction factor } \\ \mathrm{g} & \text { gravity }\left(\mathrm{m} / \mathrm{s}^{2}\right) \\ \mathrm{Gr}_{\mathrm{g}} & \text { gravitational Grashof number } \\ \mathrm{Gr} \Omega & \text { rotational Grashof number } \\ \mathrm{h} & \text { convective heat transfer coefficient }\left(\mathrm{W} / \mathrm{m}^{2}-\mathrm{K}\right) \\ \mathrm{L} & \text { Length of the pipe }(\mathrm{m}) \\ \mathrm{N} & \text { rotation rate } \\ \mathrm{Nu} & \text { average Nusselt number } \\ \mathrm{P} & \text { Pressure }(\mathrm{Pa}) \\ \mathrm{Pe} & \text { Peclet number } \\ \mathrm{Pr} & \text { Prandtl number } \\ \mathrm{q}_{\mathrm{w}} & \text { heat flux }\left(\mathrm{W} / \mathrm{m}^{2}\right) \\ \mathrm{Re}_{\mathrm{a}} & \text { axial Reynolds number } \\ \mathrm{Re}_{\Omega} & \text { rotational Reynolds number } \\ \mathrm{k} & \text { thermal conductivity }(\mathrm{W} / \mathrm{m}-\mathrm{K}) \\ \mathrm{T} & \text { Temperature }(\mathrm{K}) \\ \mathrm{V}_{\mathrm{r}} & \text { r component of velocity }(\mathrm{m} / \mathrm{s}) \\ \mathrm{V}_{\theta} & \theta \text { component of velocity }(\mathrm{m} / \mathrm{s}) \\ \mathrm{V}_{\mathrm{z}} & \mathrm{z} \text { component of velocity }(\mathrm{m} / \mathrm{s}) \\ \mathrm{V}_{\mathrm{m}} & \text { mean flow velocity }(\mathrm{m} / \mathrm{s})\end{array}$

Greek symbols$$
\rho
$$$$
\mu
$$$$
\beta
$$$$
\Omega
$$$$
v
$$

\section{REFERENCES}

Abotsi, O. Y., and Kizito, J. P., 2020, "Numerical study of heat transfer augmentation in an axially rotating pipe equipped with Kenics mixer," Case Studies in Thermal Engineering, 100695.

https://doi.org/10.1016/j.csite.2020.100695

Borisenko, A. I., Kostikov, O. N., and Chumachenko, V. I., 1973, "Experimental study of turbulent flow in a rotating channel," Journal of engineering physics, 24(6), 770-773. https://doi.org/10.1007/BF00831681

Bradshaw, P.,1969, "The analogy between streamline curvature and buoyancy in turbulent shear flow," Journal of Fluid Mechanics, 36(1), 177-191.

https://doi.org/10.1017/S0022112069001583

Cannon, J. N., and Kays, W. M.,1969, "Heat Transfer to a Fluid Flowing Inside a Pipe Rotating About Its Longitudinal Axis," ASME. J. Heat Transfer. 91(1): 135-139.

https://doi.org/10.1115/1.3580069

Chong, Y. C., 2015, "Thermal analysis and airflow modeling of electrical machines," (Ph.D. Dissertation, University of Edinburgh)

Faghri, A., Gogineni, S., \& Thomas, S., 1993, "Vapor flow analysis of an axially rotating heat pipe" International Journal of Heat and Mass Transfer, 36(9), 2293-2303. https://doi.org/10.1016/S0017-9310(05)80114-0

Gai, Y., Kimiabeigi, M., Chong, Y. C., Goss, J., Kulan, M. C., Widmer, J. D., Steven, A., and Staton, D. A.,2018, "Pressure Loss Modelling in a Water-Cooled Hollow-Shaft Rotor for an Automotive Traction Motor," 2018 XIII International Conference on Electrical Machines (ICEM), Alexandroupoli, 2018, pp. 1297-1302. https://doi.org/10.1109/ICELMACH.2018.8506852

Gai, Y., Kimiabeigi, M., Widmer, J. D., Chong, Y. C., Goss, J., SanAndres, U., and Staton, D. A., 2017, "Shaft cooling and the influence on the electromagnetic performance of traction motors," In 2017 IEEE International Electric Machines and Drives Conference (pp. 1-6). https://doi.org/10.1109/IEMDC.2017.8002307

Granados-Ortiz, F. J., Ortega-Casanova, J., and Lai, C. H., 2019, “Twostep numerical simulation of the heat transfer from a flat plate to a swirling jet flow from a rotating pipe," International Journal of Numerical Methods for Heat \& Fluid Flow.

https://dx.doi.org/10.1108/HFF-04-2019-0343

Hettegger, M., Streibl, B., Biro, O., and Neudorfer, H., 2011, "Measurements and simulations of the convective heat transfer coefficients on the end windings of an electrical machine," IEEE Transactions on Industrial Electronics, 59(5), 2299-2308. https://doi.org/10.1109/TIE.2011.2161656

Hirai, S., Takagi, T., and Matsumoto, M.,1988, "Predictions of the Laminarization Phenomena in an Axially Rotating Pipe Flow" ASME. $J$. Fluids Eng. 110(4): 424-430. https://doi.org/10.1115/1.3243573

Imao, S., Itoh, M., Yamada, Y., and Zhang, Q., 1992, “The characteristics of spiral waves in an axially rotating pipe," Experiments in Fluids, 12(45), 277-285.

https://doi.org/10.1007/BF00187306 
Imao, S., Itoh, M., and Harada, T., 1996, "Turbulent characteristics of the flow in an axially rotating pipe," International journal of heat and fluid flow, 17(5), 444-451. https://doi.org/10.1016/0142-727X(96)00057-4

Kikuyama, K., Murakami, M., and Nishibori, K., 1983a, "Development of Three-Dimensional Turbulent Boundary Layer in an Axially Rotating Pipe,” ASME. J. Fluids Eng. 105(2): 154-160.

https://doi.org/10.1115/1.3240955

Kikuyama, K., Murakami, M., Nishibori, K., and Maeda, K., 1983b, "Flow in an Axially Rotating Pipe: A calculation of flow in the saturated region," Bulletin of JSME, 26(214), 506-513. https://doi.org/10.1299/jsme1958.26.506

Lian, W., Sun, Z., Han, T., and Xuan, Y, 2020, "Experiment study on the boiling heat transfer of liquid film in a rotating pipe.," Frontiers in Heat and Mass Transfer (FHMT), 14, 10

http://dx.doi.org/10.5098/hmt.14.10

Mackrodt, P. A.,1976, "Stability of Hagen-Poiseuille flow with superimposed rigid rotation," Journal of Fluid Mechanics, 73(1), 153164.

https://doi.org/10.1017/S0022112076001304

Murakami, M., and Kikuyama, K.,1980, "Turbulent Flow in Axially Rotating Pipes,” ASME. J. Fluids Eng. 102(1): 97-103.

https://doi.org/10.1115/1.3240633

Nagib, H. M., Lavan, Z., Fejer, A. A., and Wolf Jr, L.,1971, "Stability of pipe flow with superposed solid-body rotation," The Physics of Fluids, 14(4), 766-768.

https://doi.org/10.1063/1.1693503

Nishibori, K., Kikuyama, K., and Murakami, M.,1987, "Laminarization of Turbulent Flow in the Inlet Region of an Axially Rotating Pipe: Fluids Engineering," JSME International Journal, 30(260), 255-262.

https://doi.org/10.1299/jsme1987.30.255

Ould-Rouiss, M., Dries, A., and Mazouz, A., 2010, "Numerical predictions of turbulent heat transfer for airflow in rotating pipe," International journal of heat and fluid flow, 31(4), 507-517. https://doi.org/10.1016/j.ijheatfluidflow.2010.02.015
Pedley, T. J.,1969, "On the instability of viscous flow in a rapidly rotating pipe," Journal of Fluid Mechanics, 35(1), 97-115. https://doi.org/10.1017/S002211206900098X

Rich, G., and Beer, H., 1989, "Fluid flow and heat transfer in an axially rotating pipe-I. Effect of rotation on turbulent pipe flow," International Journal of heat and mass transfer, 32(3), 551-562. https://doi.org/10.1016/0017-9310(89)90143-9

Seghir-Ouali, S., Saury, D., Harmand, S., Phillipart, O., and Laloy, D.,2006, "Convective heat transfer inside a rotating cylinder with an axial airflow," International Journal of thermal sciences, 45(12), 11661178. https://doi.org/10.1016/j.ijthermalsci.2006.01.017

Shchukin, V. K., 1967, "Hydraulic resistance of rotating tubes," Journal of engineering physics, 12(6), 418-422. https://doi.org/10.1007/BF00832650

Satake, S., Kunugi, T., 2002, "Direct numerical simulation of turbulent heat transfer in an axially rotating pipe: Reynold stress and scalar-flux budgets." Int. J. Numer. Methods Heat Fluid Flow 12 (8), 985-1008. https://doi.org/10.1108/09615530210448723

Staton, D. A., and Cavagnino, A.,2008, "Convection heat transfer and flow calculations suitable for electric machines thermal models," IEEE transactions on industrial electronics, 55(10), 3509-3516. https://doi.org/10.1109/TIE.2008.922604

Weigand, B., and Beer, H., 1992, "Fluid flow and heat transfer in an axially rotating pipe subjected to external convection." International journal of heat and mass transfer, 35(7), 1803-1809. https://doi.org/10.1016/0017-9310(92)90151-H

Weigand, B., and Beer, H., 1994, "On the universality of the velocity profiles of a turbulent flow in an axially rotating pipe," Applied scientific research, 52(2), 115-132. https://doi.org/10.1007/BF00868054

White, A., 1964, "Flow of a Fluid in an Axially Rotating Pipe," Journal of Mechanical Engineering Science, 6(1), 47-52. https://doi.org/10.1243/JMES JOUR 196400601002 